INPLASY

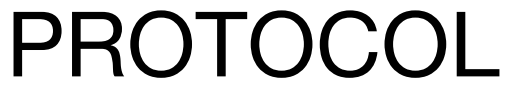

To cite: Woolley et al. Does oil pulling with coconut oil improve oral health and dental hygiene? A protocol of a systematic review. Inplasy protocol 202060084. doi: 10.37766/inplasy2020.6.0084

Received: 22 June 2020

Published: 22 June 2020

Corresponding author: Julian Woolley

julianwoolley@gmail.com

Author Affiliation:

King's College Dental Hospital

Support: None

Review Stage at time of this submission: Preliminary searches.

Conflicts of interest: None.

\section{Does oil pulling with coconut oil improve oral health and dental hygiene? A protocol of a systematic review}

Woolley, J1; Gibbons, T2; Patel, K³ Sacco, R4.

Review question / Objective: Is there sufficient evidence that coconut oil when used in an oil pulling technique improves dental hygiene and oral health compared to other conventional and evidence-based interventions? Population (P): any human participant; Intervention (I): oil pulling with coconut oil; Comparison (C): conventional oral hygiene routines and alternative evidence-based interventions; Outcome (0): effect on oral hygiene and dental hygiene.

Condition being studied: Oral health and dental hygiene. Information sources: The following six databases will be explored: PubMed, Medline, EMBASE, AMED, CENTRAL and CINAHL. The authors of any studies eligible for inclusion with insufficient information will be contacted directly by email.

INPLASY registration number: This protocol was registered with the International Platform of Registered Systematic Review and Meta-Analysis Protocols (INPLASY) on 22 June 2020 and was last updated on 22 June 2020 (registration number INPLASY202060084).

\section{INTRODUCTION}

Review question / Objective: Is there sufficient evidence that coconut oil when used in an oil pulling technique improves dental hygiene and oral health compared to other conventional and evidence-based interventions? Population ( $P$ ): any human participant; Intervention (I): oil pulling with coconut oil; Comparison (C): conventional oral hygiene routines and alternative evidence-based interventions; Outcome (O): effect on oral hygiene and dental hygiene.

Rationale: The rationale of this review is to appraise all data from randomized controlled trials to determine whether there 
is sufficient evidence that coconut oil when used in an oil pulling technique improves dental hygiene and oral health compared to other conventional and evidence-based interventions.

Condition being studied: Oral health and dental hygiene.

\section{METHODS}

Search strategy: The following six databases will be explored: PubMed, Medline, EMBASE, AMED, CENTRAL and CINAHL to locate all relevant and potential randomized controlled trials. A search strategy has been developed: 1 . Periodontal [MeSH terms] or Periodont [MeSH terms] or Periodontitis [MeSH terms] or Gingivitis [MeSH terms] or Gingival [MeSH terms] or Periodontal disease [MeSH terms] or Periodontics [MeSH terms] or Oral [MeSH terms] or Dental [MeSH terms] or Oral health [MeSH terms] or Oral hygiene [MeSH terms] or Dental hygiene [MeSH terms] or Hallitosis 2. Coconut pulling [MeSH terms] or Coconut oil [MeSH terms] or Oil pulling [MeSH terms] or Ayurveda [MeSH terms] or Ayurvedic medicine 3. \#1 and \#2 The search strategy will include appropriate changes in the keywords and follow syntax rules for each of the six databases.

Participant or population: Human participants with no restriction of age, gender, sample size or ethnic origin.

Intervention: Oil pulling with coconut oil.

Comparator: All participants who underwent any other interventions.

Study designs to be included: Randomized controlled trials.

Eligibility criteria: Published or unpublished randomised controlled trials. Papers will be obtained prior to June 2020 and no restriction will be placed on initial date of publication.

Information sources: The following six databases will be explored: PubMed,
Medline, EMBASE, AMED, CENTRAL and CINAHL. The authors of any studies eligible for inclusion with insufficient information will be contacted directly by email.

Main outcome(s): The primary outcome is to determine whether oil pulling with coconut oil improves oral health.

Additional outcome(s): The secondary outcomes are to determine whether the duration of use and method of delivery of coconut oil affect oral health and dental hygiene. In addition; to compare this to alternative conventional interventions.

Data management: Following full-text screening, data extraction will be completed using a predefined and standardized Microsoft Excel form by two investigators independently. Any discrepencies will be solved using a third investigator through discusssion.

Quality assessment / Risk of bias analysis: Two authors will independently appraise the risk of bias in this review. The Cochrane Handbook for Systematic Reviews of Interventions will be used to appraise the risk for each randomized controlled trial. Quality assessment will be carried using the JADAD score for each study and graded using the Oxford Center of Evidence-Based-Medicine: Levels of Evidence. A third author will resolve, by discussion, any conflicts arising between the first two authors.

Strategy of data synthesis: Following a comprehensive screening to determine eligible studies, all selected papers will be carefully read to identify author(s); year of publication; study design; population sample, interventions and oral hygiene adjustments. To assess our outcomes, data will be subsequently extracted from each study and analysed by a number of authors. Where pooling is inappropriate, the results of the trials will be reported as a narrative description using detailed commentary on the study findings, interventions and controls and outcomes. 
Subgroup analysis: All participants will be included in the final statistical analysis. If data permits, we will perform a subgroup analysis.

Sensibility analysis: If sufficient available data are extracted, we will plan to conduct sensitivity analysis to check the stability for the outcome results by excluding low methodological quality studies.

Language: No language restrictions will be applied.

Country(ies) involved: England.

Keywords: Ayurvedic medicine, coconut oil, oil pulling, oral health, dental hygiene.

Dissemination plans: We will submit this study to a peer-reviewed journal for publication.

Contributions of each author:

Author 1 - Julian Woolley.

Author 2 - Tatjana Gibbons.

Author 3 - Kajal Patel.

Author 4 - Roberto Sacco. 